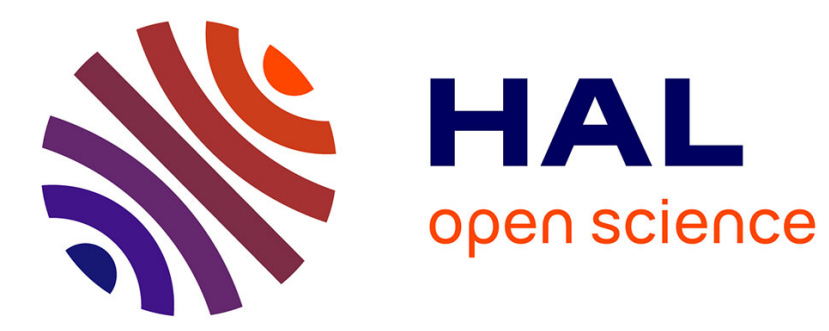

\title{
Production and dissipation of turbulent fluctuations close to a stagnation point
}

Peter Huck, Nathanaël Machicoane, Romain Volk

\section{To cite this version:}

Peter Huck, Nathanaël Machicoane, Romain Volk. Production and dissipation of turbulent fluctuations close to a stagnation point. Physical Review Fluids, 2017, 2, 10.1103/PhysRevFluids.2.084601 . hal-02519231

\section{HAL Id: hal-02519231 \\ https://hal.science/hal-02519231}

Submitted on 25 Mar 2020

HAL is a multi-disciplinary open access archive for the deposit and dissemination of scientific research documents, whether they are published or not. The documents may come from teaching and research institutions in France or abroad, or from public or private research centers.
L'archive ouverte pluridisciplinaire HAL, est destinée au dépôt et à la diffusion de documents scientifiques de niveau recherche, publiés ou non, émanant des établissements d'enseignement et de recherche français ou étrangers, des laboratoires publics ou privés. 


\title{
Production and dissipation of turbulent fluctuations close to a stagnation point
}

\author{
Peter D. Huck, ${ }^{1}$ Nathanaël Machicoane, ${ }^{2}$ and Romain Volk ${ }^{1, *}$ \\ ${ }^{1}$ Université de Lyon, ENS de Lyon, Université Claude Bernard, CNRS, Laboratoire de Physique, F-69342 \\ Lyon, France \\ ${ }^{2}$ Department of Mechanical Engineering, University of Washington, Seattle, Washington 98195, USA
}

(Received 12 January 2017; published 4 August 2017)

\begin{abstract}
In this article, we investigate the production and dissipation of turbulence in a region where the mean flow topology presents a stagnation point. Our goal is to understand the generation of anisotropic fluctuations and their influence on production, dissipation, and transport of turbulent kinetic energy. In order to investigate the local turbulent kinetic energy budget, we use a shadow particle tracking velocimetry technique (S-PTV) to track Lagrangian tracers in a large portion of a turbulent von Kármán flow produced by counterrotating disks. We observe that the flow produced in a square tank is bistable, with each of the two states resembling impinging jets. This stagnation-point topology is responsible for the strong anisotropy of velocity fluctuations observed in these type of flows. The production of turbulence locally exceeds the dissipation rate. As a consequence, the flow is to be considered as strongly inhomogeneous as the fluxes of turbulent kinetic energy are non-negligible when compared to the production and dissipation terms.
\end{abstract}

DOI: 10.1103/PhysRevFluids.2.084601

\section{INTRODUCTION}

A large part of turbulence theory has been developed in the context of homogeneous and isotropic turbulence [1]. In such situations, the mean flow is considered to be zero and flow properties are assumed to be invariant in space. However, common turbulent flows are often produced by mechanical forcing in the presence of boundaries, leading to nonzero mean flows with complex topologies. Such is the case of a mechanically forced flow in a closed volume, which results in regions dominated by shear or recirculations with hyperbolic points. In this context, shear flows are well documented [2,3], but the action of strain has received less attention and our knowledge relies either on measurements performed in quasihomogeneous situations [4,5] or in near-wall regions [6].

We address the production and dissipation of turbulent fluctuations experimentally using a von Kármán flow with a square cross section (described in Sec. II). A mean flow is produced by counter-rotating impellers which impose inertial steering on the fluid and result in strong azimuthal velocity gradients, and it is generally considered to give rise to shear-driven turbulence. However, the central region's stagnation point is typified by a strong straining motion, which is an often overlooked aspect of this configuration. We investigate the role of this topology in turbulence production; in particular, we address the origin of the anisotropic velocity fluctuations observed in these type of flows [7-9].

The experiment is performed in a large volume in the central region where Lagrangian tracers are tracked and all scales of their motions are resolved. We show that the flow is bistable (Sec. III), where each of the two states resembles a pair of impinging jets with one stable and two unstable directions. This situation is not observed to be "free-shear flow" turbulence, but rather its main features point strongly to a stagnation-point topology whose study is lacking in the literature.

The local turbulent kinetic energy budget is presented (Sec. IV) where the quantity $\left\langle\vec{a}^{\prime} \cdot \vec{v}^{\prime}\right\rangle=$ $\langle\vec{a} \cdot \vec{v}\rangle-\langle\vec{a}\rangle \cdot\langle\vec{v}\rangle$ is shown to be an accurate local measurement of the dissipation in the vicinity of

*romain.volk@ens-lyon.fr 

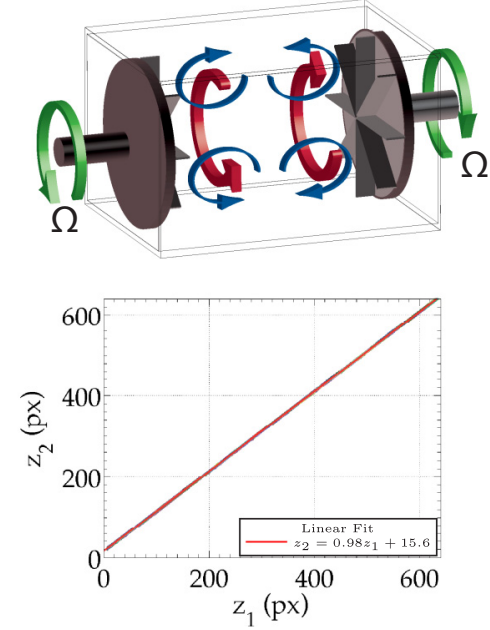

(a)

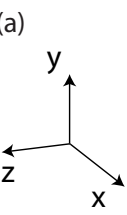

(c) (b)

Cam 1

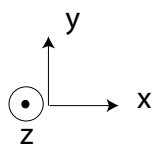

L1
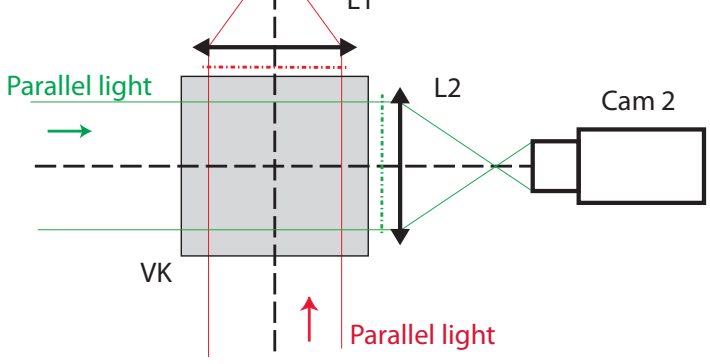

FIG. 1. (a) Sketch of the counter-rotating von Kármán flow. Arrows indicate the topology of the mean flow when averaged over both states. (b) Optical setup for S-PTV with two identical optical arrangements forming an angle $\theta=90^{\circ}$ : parallel light is propagating through the flow volume before being collected using a 15-cm-diameter lens whose function is to redirect the light into the camera objective of the camera. The optical system $\left[L_{2}+\right.$ objective] is focused on the output face of the vessel marked with a dash-dotted line. (c) Raw axial position of particles, $z_{2}$ (in pixels), as measured on cam 2 as a function of $z_{1}$ measured on cam 1 . The line corresponds to a linear fit $z_{2}=a z_{1}+b$ with $a=0.98, b=15.6$ pixels.

the stagnation point. We demonstrate a mechanism by which energy is efficiently extracted from the mean flow such that production of turbulence is twice the value of energy dissipation. As a result, a significant amount of energy is transported by turbulent fluctuations in the vicinity of the stagnation point and renders the flow spatially inhomogeneous.

\section{EXPERIMENTAL SETUP}

\section{A. Description of the flow}

The experimental apparatus is a von Kármán flow identical to the one used in Ref. [10]. A water-Ucon ${ }^{\mathrm{TM}}$ mixture with viscosity 8.2 times that of water and equal density is used to fill a square cylinder where the flow is produced using two bladed discs of diameter $R=7.1 \mathrm{~cm}$ that counter-rotate at constant frequency $\Omega$ [Fig. 1(a)] and are separated by $20 \mathrm{~cm}$ while the tank width is $15 \mathrm{~cm}$. The angular velocity of the disks is adjusted so that they rotate at same velocity, but in opposite directions, imposing an inertial forcing which generates a fully turbulent flow $\left(\operatorname{Re}_{\lambda} \sim 190\right)$. As opposed to wind tunnel flows, the von Kármán flow has a mean tridimensional spatial structure and is sketched in Fig. 1(a). The disks rotate in opposite directions and generate a large azimutal velocity component of order $2 \pi R \Omega$ which goes to zero in the midplane $(z=0)$ of the square tank. The presence of blades improves stirring and creates an intense poloidal recirculation with a stagnation point in the geometrical center of the vessel. The dominant flow characteristics in the central region are strong shear and intense and anisotropic fluctuations close to the stagnation point [7-9], where most of the dissipation occurs $[11,12]$. Table I gives a summary of the flow parameters measured at the stagnation point of the present setup.

\section{B. Three-dimensional-particle tracking setup}

We perform particle tracking of Lagrangian tracers (250- $\mu$ m polystyrene particles, less than three times the Kolmogorov scale) in a large volume $6 \times 6 \times 5.5 \mathrm{~cm}^{3}$ centered around the geometrical 
TABLE I. Parameters of the flow. $\Omega$, rotation rate of the discs. The rms velocities are obtained at the geometrical center of the flow using data points located in a sphere with a $1-\mathrm{cm}$ radius. The kinematic viscosity of the water-Ucon ${ }^{\mathrm{TM}}$ mixture is $v=8.210^{-6} \mathrm{~m}^{2} \mathrm{~s}^{-1}$ with a density $\rho=1000 \mathrm{~kg} \mathrm{~m}^{-3}$. The dissipative time scale is estimated from the zero crossing $\left[t_{0}=\left(t_{0 x}+t_{0 y}+t_{0 z}\right) / 3\right]$ of each component in the acceleration autocorrelation function $t_{0} \simeq 2.2 \tau_{\eta}[9,14,15]$, the dissipation rate is estimated as $\varepsilon=v / \tau_{\eta}^{2}$, dissipative length scale is $\eta=\left(v^{3} / \varepsilon\right)^{1 / 4}$, and the Taylor-based Reynolds number is estimated as $\operatorname{Re}_{\lambda}=\sqrt{15 u^{\prime 4} / \nu \varepsilon}$ with $u^{\prime}=$ $\sqrt{\left(u_{x}^{\prime 2}+u_{y}^{\prime 2}+u_{z}^{\prime 2}\right) / 3}$. The large-scale Reynolds number is $\operatorname{Re}=2 \pi R^{2} \Omega / \nu$.

\begin{tabular}{|c|c|c|c|c|c|c|c|c|c|c|}
\hline $\begin{array}{l}\Omega \\
\mathrm{Hz}\end{array}$ & State & $\begin{array}{c}u_{x}^{\prime} \\
\mathrm{ms}^{-1}\end{array}$ & $\begin{array}{c}u_{y}^{\prime} \\
\mathrm{ms}^{-1}\end{array}$ & $\begin{array}{c}u_{z}^{\prime} \\
\mathrm{ms}^{-1}\end{array}$ & $\begin{array}{c}u^{\prime} \\
\mathrm{ms}^{-1}\end{array}$ & $\begin{array}{c}\tau_{\eta} \\
\mathrm{ms}\end{array}$ & $\begin{array}{c}\eta \\
\mu \mathrm{m}\end{array}$ & $\begin{array}{c}\varepsilon \\
\mathrm{Wkg}^{-1}\end{array}$ & $R_{\lambda}$ & $\operatorname{Re}$ \\
\hline \multirow{2}{*}{4.2} & $x$ dominant & 0.45 & 0.29 & 0.25 & 0.34 & 2.9 & 154 & 1.0 & 155 & 16200 \\
\hline & Averaged & 0.39 & 0.37 & 0.24 & 0.34 & 2.9 & 154 & 1.0 & 155 & 16200 \\
\hline \multirow[t]{2}{*}{5.5} & $x$ dominant & 0.58 & 0.39 & 0.33 & 0.45 & 2.0 & 128 & 2.1 & 190 & 21200 \\
\hline & Averaged & 0.50 & 0.49 & 0.33 & 0.45 & 2.0 & 128 & 2.1 & 190 & 21200 \\
\hline \multirow[t]{2}{*}{6.9} & $x$ dominant & 0.74 & 0.48 & 0.41 & 0.56 & 1.5 & 111 & 3.6 & 225 & 26700 \\
\hline & Averaged & 0.62 & 0.62 & 0.41 & 0.56 & 1.5 & 111 & 3.6 & 225 & 26700 \\
\hline
\end{tabular}

center $(x, y, z)=(0,0,0)$ of the flow. Tracers are tracked with two high-speed video cameras (Phantom V.12, Vision Research, 1 Mpixel at $7 \mathrm{kHz}$ ) with a resolution of $800 \times 768$ pixels and a high frame rate of $f_{s}=12 \mathrm{kHz}$, chosen to adequately resolve particle acceleration. The camera arrangement, inspired by previous work [10], is depicted in Fig. 1(b). It consists of two arms forming an angle $\theta=90^{\circ}$ with parallel lighting. This large parallel ray $(15 \mathrm{~cm}$ in diameter $)$ intersects the flow volume before being collected onto the camera using a doublet consisting of a large lens $(15 \mathrm{~cm}$ in diameter, with a 50-cm focal length) and the camera objective. The doublet is focused on the face of the tank closest to the camera which was found to be the configuration best adapted to tracking small objects. As this arrangement requires precision mounting, all optical elements are aligned using large, homemade reticules also used to measure the magnification in each arm. When placing an object in the field of view, it appears as a black shadow on a white background corresponding to the parallel projection of the object on the sensor. As opposed to conventional PTV, particles are tracked independently on each view prior to stereo matching so that camera 1 provides the $\left(x_{1}, z_{1}\right)$ positions while camera 2 measures their $\left(y_{2}, z_{2}\right)$ positions [13]. The $z$ coordinates are redundant and an affine relationship $\left[z_{2}=a z_{1}+b\right.$, Fig. 1(c)] permits the stereo matching of trajectories satisfying $\max \left(\left|z_{2}(t)-a z_{1}(t)-b\right|\right)<3$ pix, which is smaller than the particle's apparent radii on the image. This affine relation is first obtained with a dilute ensemble of particles that permits easy stereo matching within a pair of movies and recursive estimations of the fit parameters $(a=0.98$, $b=15.6$ pix is used here). Together with the magnification of camera 1 (90 $\mu \mathrm{m}$ per pixel), the calibration provides all requisite information concerning particle positions in laboratory coordinates. As the experiment was run at a low particle concentration, each pair of movies leads to an ensemble of trajectories from which single-particle statistics can be computed. For each Reynolds number considered, we record 500 pairs of movies lasting $1.3 \mathrm{~s}$, each pair leading to $\mathcal{O}(1000)$ trajectories with a mean duration $\langle t\rangle \sim 0.25 / \Omega$. A large ensemble of $\mathcal{O}\left(4 \times 10^{5}\right)$ trajectories allows Lagrangian single-particle statistics to be conditioned in space in order to investigate the nonhomogeneity of the flow properties.

\section{BISTABILITY AND MEAN FLOW PROPERTIES}

\section{A. Mean flow topology}

The counter-rotating von Kármán flow is known to exhibit long time dynamics [16]. To investigate the stationarity of the flow, we conducted 8-h measurement campaigns, obtaining 100 films separated by $5 \mathrm{~min}$ of data transfer. Figures 2(a) and 2(b) display the time evolution of the rms values of the 
(a)

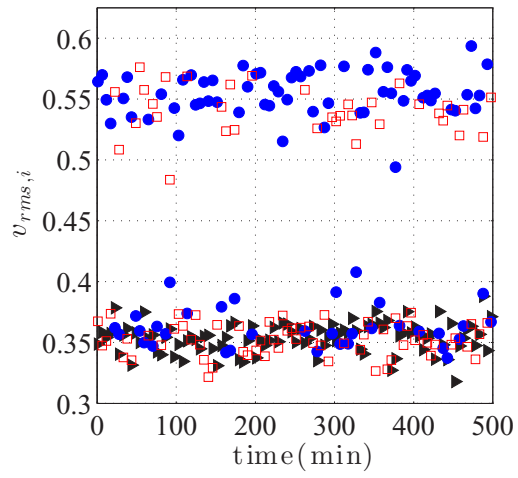

(c)

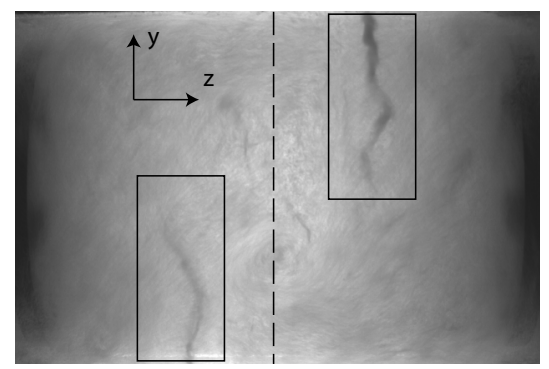

(b)

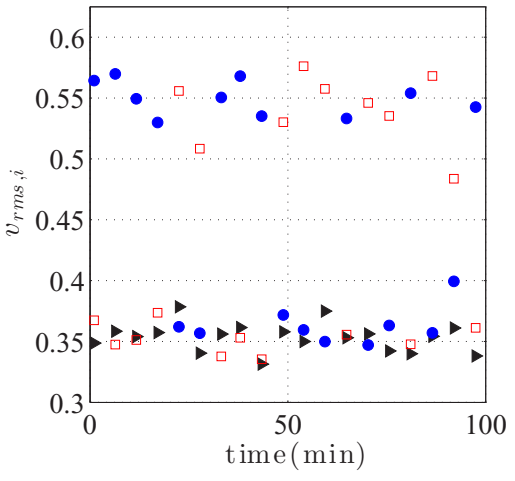

(d)

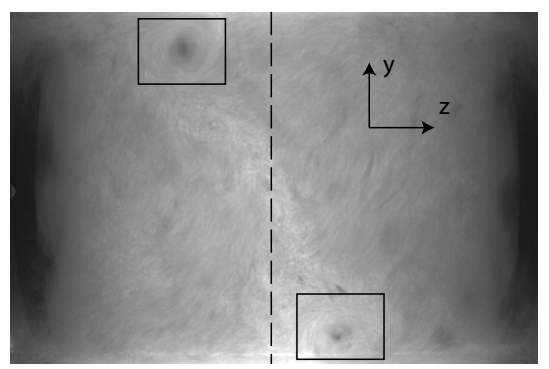

FIG. 2. Panels (a) and (b): Time evolution of the rms value of the velocity computed from each movie at $\operatorname{Re}_{\lambda}=190$. The time is defined as $t=k T$ where $k$ is the number of the movie and $T=5$ min is the time between each successive recordings. $\bullet, x$ component; $\square, y$ component; and $\triangleright, z$ component. Panels (c) and (d): Backlight visualization of bubbles injected in the flow, and dark regions corresponding to bubbles concentrated in vortices are marked with a box. The webcam and the LED panel are placed apart the vessel in the $x$ direction, with the disks being visible on the sides of each image. (c) System of two vertical vortices aligned with the $y$ direction. (d) System of two horizontal vortices aligned with the $x$ direction. In all cases, the shear layer is located at the midplane (marked with a dashed line).

velocity components using all trajectories in each pair of movies for the intermediate Reynolds number and are computed directly from the trajectories by

$$
v_{\mathrm{rms}, i}(t)=\sqrt{\frac{1}{N_{p}} \sum_{p \in \text { movie }}^{N_{p}}\left[\overline{\left(v_{i}^{p}\right)^{2}}-\left(\overline{\left.v_{i}^{p}\right)^{2}}\right]\right.}, \quad i=x, y, z,
$$

where $N_{p}$ is the total number of particle trajectories $p$ in the $k$ th pair of movies (leading to $t=5 k$, in minutes), and $\overline{v_{i}^{p}}$ is the velocity component $i=x, y, z$ averaged in time over its duration $T_{p}$. We observe that the axial component $\left(v_{\mathrm{rms}, z}\right)$ is always close to $0.35 \mathrm{~m} \mathrm{~s}^{-1}$ while the transverse components ( $v_{\mathrm{rms}, x}$ and $\left.v_{\mathrm{rms}, y}\right)$ alternate between two values: 0.35 and $0.55 \mathrm{~m} \mathrm{~s}^{-1}$. Moreover, $v_{\mathrm{rms}, x}$ and $v_{\text {rms, } y}$ never have a large value at the same time, but instead exchange values. This behavior is observed regardless of the rotation frequency in the fully turbulent regime, indicating that the large-scale flow is bistable with one transverse component dominating the other. The two states of the flow can be directly observed by introducing bubbles into the apparatus and using a backlight configuration with a webcam operating at $15 \mathrm{~Hz}$ oriented in the $x$ direction to observe a field lit by a LED panel. Upon visualization, coherent vortex structures trap the lighter bubble phase and stand out clearly in the images [Figs. 2(c) and 2(d)]. These vortices occur in pairs attached to either set of horizontal [Fig. 2(c)] or vertical [Fig. 2(d)] walls and are situated at equal distance from the 


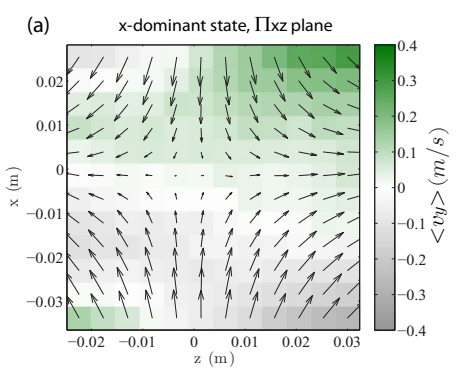

(d) $\quad y$-dominant state, $\Pi x z$ plane

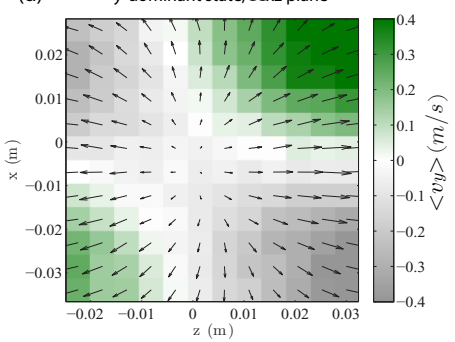

(b)

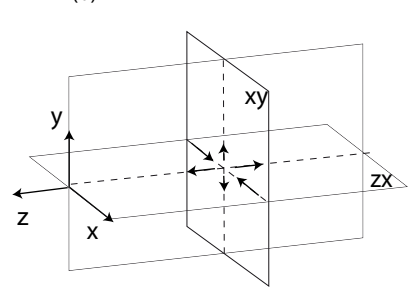

(c) x-dominant state, Пxy plane

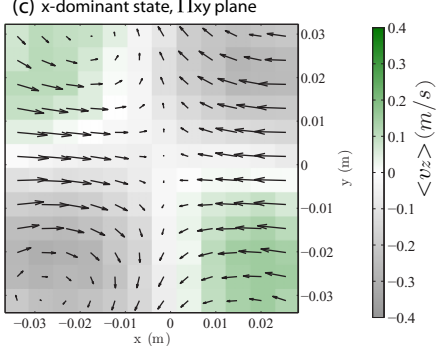

(e)

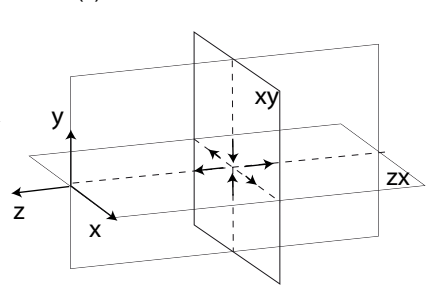

(f) y-dominant state, Пxy plane

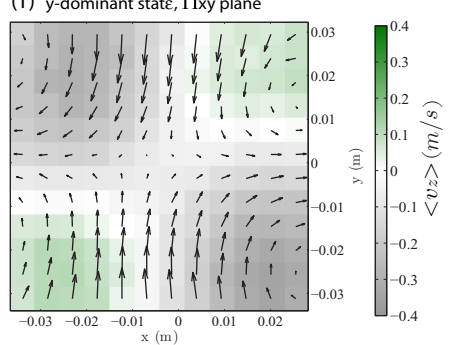

FIG. 3. (a) $\Pi_{x z}=(x, y=0, z)$ cut of the reconstructed Eulerian mean velocity field of the $x$-dominant state. Arrows are $\left(\left\langle v_{x}\right\rangle,\left\langle v_{z}\right\rangle\right)$; the color coding is for the $\left\langle v_{y}\right\rangle$. (b) Topology of the $x$-dominant state at the geometric center. (c) $\Pi_{x y}=(x, y, z=0)$ cut of the $x$-dominant state. Arrows are $\left(\left\langle v_{x}\right\rangle,\left\langle v_{y}\right\rangle\right)$; the color coding is for the $\left\langle v_{z}\right\rangle$. Panels (d)-(f): Same figures as panels (a)-(c) for the $y$-dominant state.

midplane, which is also the location of the shear layer. The vortices have their own dynamics. Their positions are observed to fluctuate and may be found nearly one vortex core away from their typical wall-anchored positions. These vortices are thought to be central to the present bistability for which the persistency of either horizontal or vertical state is much longer (several minutes, confirmed by laser Doppler velocimetry measurements) than the large eddy turnover time of $1 / \Omega \simeq 0.2 \mathrm{~s}$. Reversals are preceded by a "dancing" motion whereby the vortex pairs become unstable and detach from their wall-anchored positions, eventually shifting $90^{\circ}$ from a vertical set to a horizontal set or vice versa. Such a scenario is specific to the square-cylinder geometry, and differs from the typical bistability observed in circular cylinders for which the two states correspond to a displacement of the shear layer and mirror each other in a reflexion about the midplane [16-18].

In order to characterize the flow properties of each state separately, the data set is separated into two ensembles with the $x$-dominant (respectively $y$-dominant) state corresponding to movies with high values of $v_{\text {rms }, x}$ (resp. $v_{\text {rms, } y}$ ). Given these sets of trajectories, one may reconstruct the mean velocity field in three dimensions $(3 \mathrm{~d}),\langle\vec{v}\rangle(x, y, z)=\left(\left\langle v_{x}\right\rangle,\left\langle v_{y}\right\rangle,\left\langle v_{z}\right\rangle\right)$, and the rms fluctuations of each velocity component. This is achieved by an Eulerian conditioning of the Lagrangian datasets on a $12^{3}$ Cartesian grid, which corresponds to a spatial resolution of $5 \mathrm{~mm}$ in each direction. We found this grid size to be sufficiently small with respect to the typical scale $(R / 2=3.5 \mathrm{~cm})$ of the flow, and sufficiently large to permit at least $\mathcal{O}(1000)$ trajectories to cross each bin, which is enough to converge both the mean and rms values of the quantities considered.

Figures 3(a) and 3(c) [respectively, Figs. 3(d) and 3(f)] display cross sections of the reconstructed mean flow of the $x$-dominant (resp., $y$-dominant) state in two perpendicular planes: the $\Pi_{x z}$ plane containing transverse and axial components, and the axially orthogonal midplane $\Pi_{x y}(x, y, z=0)$. These figures demonstrate that neither state presents a mean structure resembling that of the schematic view in Fig. 1(a), nor do they contain the double contracting axes $(x, y)$ and a single diverging direction $(z)$ near the geometrical center. Considering the $\Pi_{x z}$ plane in the $x$-dominant state, stagnation-point topology is evident and indeed a single converging direction $(x)$ is present [Fig. 3(a)]. However, the same plane in the $y$-dominant state gives two diverging axes [Fig. 3(d)]. In 
fact, these two figures would be exchanged when presenting cross sections in the $\Pi_{y z}$ plane instead of $\Pi_{x z}$ to properly account for the dominant converging direction. This reversal is an important signature of the bistability in the mean flow and is apparent when observing cross sections in the $\Pi_{x y}$ plane, which illustrate dominant components coinciding invariably with a contracting direction, similar to the topology of two impinging jets [Figs. 3(b) and 3(e)]. The presence of two unstable directions and one stable direction is confirmed when computing the (normalized) gradient tensor of the mean flow components and the Reynolds stress tensor at the origin $(x, y, z)=(0,0,0)$ in the $x$-dominant state:

$$
\begin{aligned}
\frac{1}{2 \pi \Omega}\left[\partial_{i}\left\langle v_{j}\right\rangle\right]=\left(\begin{array}{ccc}
-0.90 & 0.18 & 0.00 \\
0.00 & 0.31 & 0.00 \\
0.08 & 0.00 & 0.60
\end{array}\right), \quad \frac{1}{2\langle k\rangle}\left[\left\langle v_{i}^{\prime} v_{j}^{\prime}\right\rangle\right]=\left(\begin{array}{ccc}
0.54 & 0.00 & 0.00 \\
0.00 & 0.27 & 0.02 \\
0.00 & 0.02 & 0.18
\end{array}\right), \\
\langle k\rangle=\left(\left\langle v_{x}^{\prime 2}\right\rangle+\left\langle v_{y}^{\prime 2}\right\rangle+\left\langle v_{z}^{\prime 2}\right\rangle\right) / 2 .
\end{aligned}
$$

The principle axes of both tensors are nearly aligned with $(x, y, z)$, indicating that strain and turbulent fluctuations are highest in the dominant contracting direction. Such a result is surprising because the flow is produced by the differential rotation of the discs and one may expect the turbulence to be of the free-shear flow type. We find that the Reynolds stress tensor is diagonal with fluctuation anisotropy coinciding with the contracting and dilating directions. The absence of cross correlation between the velocity components indicates that the flow is not of the free-shear flow type [3], but is typical of stagnation-point turbulence. All of the aforementioned results are valid for the $y$-dominant state (exchanging contraction along $x$ with $y$ ) and all of the Reynolds numbers investigated. In what follows, we will consider the $x$-dominant state and the role of stagnation-point topology in the production, dissipation, and transport of turbulent kinetic energy.

\section{TURBULENT KINETIC ENERGY BUDGET (TKE)}

\section{A. Velocity fluctuations}

Velocity fluctuations in the central region are strongly anisotropic and nonhomogeneous in space, as illustrated in Fig. 4(a), which displays the averaged kinetic energy of the fluctuations, $\langle k\rangle=\left(\left\langle v_{x}^{\prime 2}\right\rangle+\left\langle v_{y}^{\prime 2}\right\rangle+\left\langle v_{z}^{\prime 2}\right\rangle\right) / 2$, in the $\Pi_{x y}$ plane. We find that the turbulent fluctuations present a local minimum at $(0,0,0)$ along the dilating direction $(x=0, y, z=0)$ and a local maximum along the contracting direction $(x, y=0, z=0)$. Velocity components along the stable direction displaying increasing anisotropy while approaching the geometric center in the $\Pi_{x y}$ plane [Fig. 4(b)], due primarily to the strong growth in dominant axis fluctuations $\left(\left\langle v_{x}^{\prime 2}\right\rangle\right)$ contrasting with the weakly varying values in the other two components.

Increasing anisotropy is understood by examining how velocity fluctuations are amplified or attenuated along a mean trajectory. Given the mean flow in the $x$-dominant state, particles starting at point $M_{0}=(x<0,0,0)$ travel directly toward the center and explore the corresponding velocity fluctuations of Fig. 4(b). In the spirit of an analysis using rapid distortion theory [19,20], we use the Reynolds decomposition $\left(v_{i}=\left\langle v_{i}\right\rangle+v_{i}^{\prime}\right)$ to write the velocity fluctuation equation:

$$
\partial_{t} v_{i}^{\prime}+\left\langle v_{j}\right\rangle \partial_{j} v_{i}^{\prime}+v_{k}^{\prime} \partial_{k}\left\langle v_{i}\right\rangle=-\frac{1}{\rho} \partial_{i} p^{\prime}+v \partial_{j} \partial_{j} v_{i}^{\prime}+v_{k}^{\prime} \partial_{k} v_{i}^{\prime}-\left\langle v_{k}^{\prime} \partial_{k} v_{i}^{\prime}\right\rangle
$$

When neglecting the right-hand side of the equation as a first approximation, this equation reads

$$
\frac{\underline{D} v_{i}^{\prime}}{\underline{D} t}=\partial_{t} v_{i}^{\prime}+\left\langle v_{j}\right\rangle \partial_{j} v_{i}^{\prime} \simeq-v_{k}^{\prime} \partial_{k}\left\langle v_{i}\right\rangle
$$

and relates the amplification of $v_{x}^{\prime}$ along a trajectory (from $x<0$ to $x=0$ ) to the velocity gradient $\left(\partial_{x}\left\langle v_{x}\right\rangle<0\right)$. Conversely, $v_{y}^{\prime}$ must decrease as $\partial_{y}\left\langle v_{y}\right\rangle>0$. Although our results are observed in a complex geometry, they are similar to findings in pioneering wind tunnel experiments [5]. It is worth mentioning that this first-order theory does not explain why the axial fluctuations only vary 
(a)

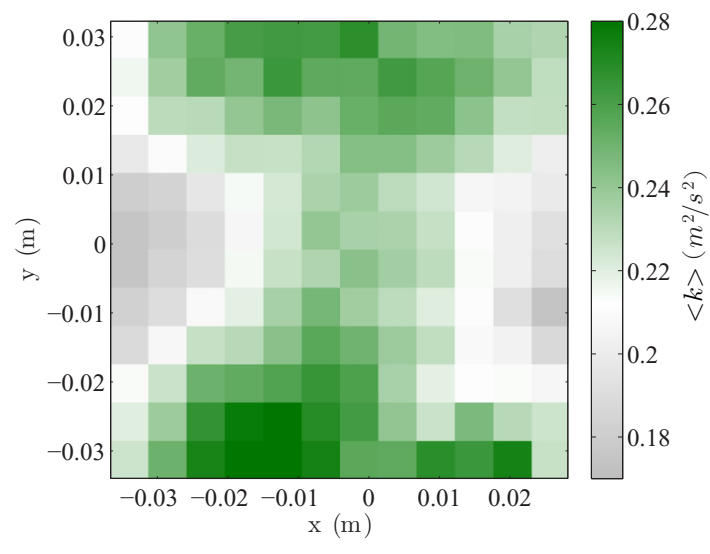

(b)

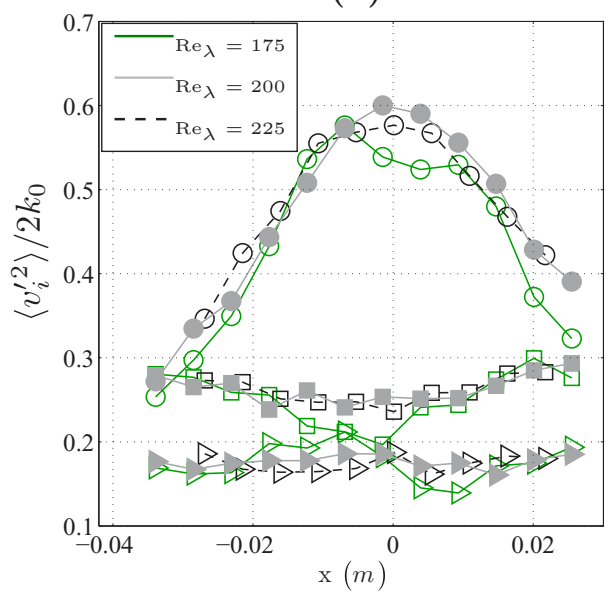

FIG. 4. (a) Section of the mean turbulent kinetic energy $\langle k\rangle=\left(\left\langle v_{x}^{\prime 2}\right\rangle+\left\langle v_{y}^{\prime 2}\right\rangle+\left\langle v_{z}^{\prime 2}\right\rangle\right) / 2$ in the plane $\Pi_{x y}=(x, y, z=0)$ measured for the $x$-dominant state at $\mathrm{Re}_{\lambda}=190$. (b) $x$ profiles of the normalized velocity components $\left\langle v_{i}^{\prime 2}\right\rangle(x, 0,0) / 2 k_{0}$, where $k_{0}=\langle k\rangle(0,0,0)$ as measured for the $x$-dominant state and the three Reynolds numbers. $\circ, x$ component; $\square, y$ component; $\triangleright, z$ component. Statistical errors of each quantity, computed from the convergence toward the mean value, are smaller than $2 \%$ of the mean. This corresponds at most to the size of the symbols on the graph.

weakly in space along $x$, which is also true along $y$ and $z$ in all the explored volume $|x, y, z|<0.4 R$. Fluctuations are not only distorted by the mean flow along particle trajectories but are also strongly dissipated close to the center in order to maintain a statistically stationary state. We turn now to a discussion of the turbulent kinetic energy balance.

\section{B. Production and dissipation of turbulence}

As previously observed, the stagnation point topology of the mean flow is responsible for the strong anisotropy of velocity fluctuations close to the geometrical center. The anisotropy is larger than in any other canonical flow (wind tunnel, channel, boundary layer, jet, or wake) and motivates the investigation of the local production, dissipation, and transport of turbulent fluctuations. A stationary, ensemble-averaged turbulent kinetic energy budget is written $[2,3]$ :

$$
\begin{aligned}
& \left\langle v_{j}\right\rangle \partial_{j}\langle k\rangle+\partial_{j}\left\langle v_{j}^{\prime} k\right\rangle=\mathcal{P}-\frac{1}{\rho} \partial_{j}\left\langle p^{\prime} v_{j}^{\prime}\right\rangle+v \partial_{j} \partial_{j}\langle k\rangle-\varepsilon . \\
& \begin{array}{llllll}
1 & \underline{2} & \underline{3} & \underline{4} & \underline{5} & \underline{6}
\end{array}
\end{aligned}
$$

This equation is established far from the propellers where no forcing term is present. Its six terms are as follows: 1 , advection of mean kinetic energy $\langle k\rangle=\left\langle v_{i}^{\prime} v_{i}^{\prime}\right\rangle / 2 ; \underline{2}$, transport of kinetic energy by turbulent fluctuations; $\underline{3}$, production of turbulent fluctuations $\overline{\mathcal{P}}=-\left\langle v_{i}^{\prime} v_{j}^{\prime}\right\rangle \partial_{i}\left\langle v_{j}\right\rangle$; $\underline{4}$, transport due to pressure velocity correlations; 5 , diffusion of mean kinetic energy; and $\underline{6}$, dissipation $\varepsilon=v\left\langle\left(\partial_{i} v_{j}^{\prime}\right)\left(\partial_{i} v_{j}^{\prime}\right)\right\rangle$. In this equation, if $v \partial_{j} \partial_{j}\langle k\rangle$ is negligible as compared to dissipation in fully turbulent flows, the pressure-velocity correlation term cannot be measured directly as it would require a measurement of pressure at the particle position. Although this correlation has been found to be much smaller than dissipation in nearly homogeneous turbulent flows [2], it is kept in the budget so that Eq. (5) is rewritten:

$$
\left\langle a_{j}^{\prime} v_{j}^{\prime}\right\rangle=\left\langle v_{j}\right\rangle \partial_{j}\langle k\rangle+\partial_{j}\left\langle v_{j}^{\prime} k\right\rangle-\mathcal{P}=-\varepsilon-\frac{1}{\rho} \partial_{j}\left\langle p^{\prime} v_{j}^{\prime}\right\rangle
$$


(a)

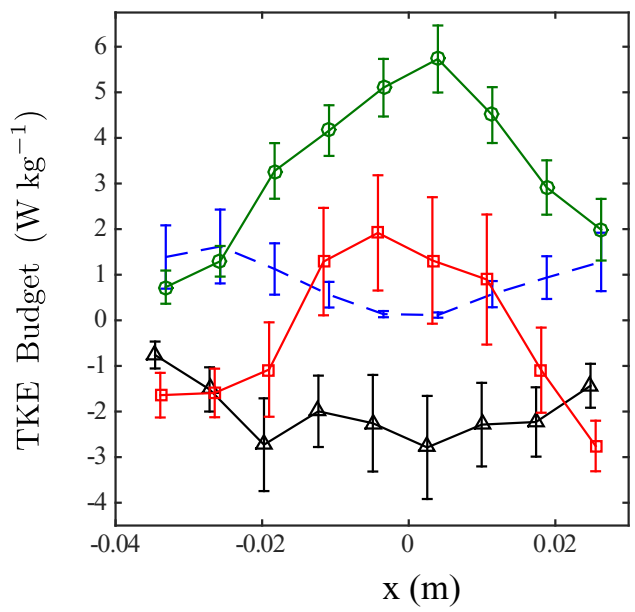

(b)

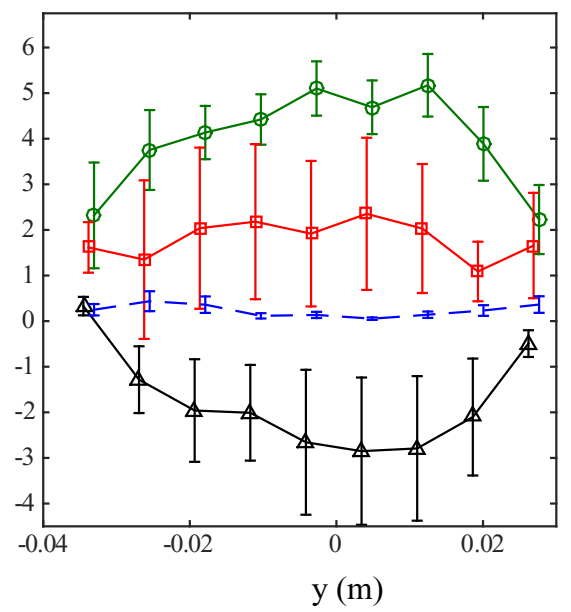

FIG. 5. (a) $x$ profiles of the different terms of Eq. (5) plotted along the line $(x, y=0, z=0)$ for the $x$-dominant state at $\operatorname{Re}_{\lambda}=190$. - - , advection of mean kinetic energy $\left\langle v_{j}\right\rangle \partial_{j}\langle k\rangle=\underline{1} ; \square$, turbulent transport term $\partial_{j}\left\langle v_{j}^{\prime} k\right\rangle=\underline{2}$; ○, production of turbulence $\mathcal{P}=-\left\langle v_{i}^{\prime} v_{j}^{\prime}\right\rangle \partial_{i}\left\langle v_{j}\right\rangle=\underline{3} ; \Delta$, averaged power per unit mass $\left\langle a_{j}^{\prime} v_{j}^{\prime}\right\rangle=\underline{4}+\underline{6}$. (b) $y$ profiles of the same quantities plotted along the line $(x=0, y, z=0)$ with the same legend. The error on both plots is given by $\pm 3 \sigma$, where $\sigma$ is defined as the standard deviation of the cumulative mean of each quantity in the range $N \in[3000,4000]$ trajectories.

where we have introduced the fluctuating acceleration $a_{j}^{\prime}=a_{j}-\left\langle a_{j}\right\rangle$. In Eq. (6), the first four terms can be computed separately by averaging the Lagrangian data in each bin using the fluctuating velocity $v_{j}^{\prime}=v_{j}-\left\langle v_{j}\right\rangle$ and acceleration $a_{j}^{\prime}=a_{j}-\left\langle a_{j}\right\rangle$, while the various mean fields are interpolated at the positions of the particles.

Figures 5(a) and 5(b) display the $x$ and $y$ profiles (in the midplane, $\Pi_{x y}$ ) of the different terms appearing in Eq. (6) obtained for the $x$-dominant state at $\operatorname{Re}_{\lambda}=190$. These profiles have been computed on a coarser Cartesian grid with $9^{3}$ bins in order to get better convergence of $\left\langle\vec{a}^{\prime} \cdot \vec{v}^{\prime}\right\rangle$ and $\left\langle\vec{v}^{\prime} k\right\rangle$ and correspond to raw data without any spatial filtering. These statistics are subject to statistical errors (in the computation of the mean of a given quantity in each bin) inherent in the limited number trajectories $[\mathcal{O}(4000)]$ passing through each bin. The error $\sigma$ is defined as the standard deviation of the cumulative mean in the range $N \in[3000,4000]$ trajectories. The figures are analyzed term by term below.

(1) Dissipation: The averaged power per unit mass $\left\langle\vec{a}^{\prime} \cdot \vec{v}^{\prime}\right\rangle$ is found to increase when approaching the center and reaches up to $-2.6 \mathrm{~W} / \mathrm{kg}$. Subtracting the mean flow contribution, $\langle\vec{a}\rangle \cdot\langle\vec{v}\rangle$, is important because $\langle\vec{a} \cdot \vec{v}\rangle$ is found to be proportional, though much larger than the nominal dissipation rate. Our calculation of local dissipation should be compared to other estimates obtained from the zero crossing of the acceleration auto-correlation function, $\tau_{0} \simeq 2.2 \tau_{\eta}[9,14,15]$, which gives $\left(\tau_{0, x}, \tau_{0, y}, \tau_{0, z}\right)=(4.7,4.4,3.9) \mathrm{ms}$, leading to $\varepsilon \in[1.8,2.6] \mathrm{W} / \mathrm{kg}$. This result holds for all bins on the Cartesian grid at each Reynolds number investigated and we conclude that $\left\langle\vec{a}^{\prime} \cdot \vec{v}^{\prime}\right\rangle$ is a reasonable estimate of the dissipation as the pressure-velocity transport term is smaller than $0.2-0.3 \varepsilon$. The latter quantity is estimated as the difference between the value of $\varepsilon$ calculated from the acceleration autocorrelation function zero crossing and local value of $\left\langle\vec{a}^{\prime} \cdot \vec{v}^{\prime}\right\rangle$.

(2) Production of turbulence: Figures 5(a) and 5(b) demonstrate that the production term is positive and locally exceeds dissipation by nearly a factor 2 close to the stagnation point. Such large production in a turbulent flow is unusual, although it has been observed for instance in a nonstationary mixing layer [21], and in the buffer layer of a channel flow [2], $\mathcal{P} \simeq \varepsilon$ is more typical in inhomogeneous flows. However, the mean flow serves to reinforce turbulent fluctuations in the $x$ 
direction where the strain is strongest, giving $\mathcal{P} \simeq-\left\langle v_{x}^{\prime 2}\right\rangle \partial_{x}\left\langle v_{x}\right\rangle-\left\langle v_{y}^{\prime 2}\right\rangle \partial_{y}\left\langle v_{y}\right\rangle-\left\langle v_{z}^{\prime 2}\right\rangle \partial_{z}\left\langle v_{z}\right\rangle \simeq$ $5 \mathrm{~W} \mathrm{~kg}^{-1}$. This dissipation deficit translates an overly efficient extraction of kinetic energy from the mean flow in the central region and requires a compensatory mechanism.

(3) Transport of turbulent fluctuations: The transport of kinetic energy has two distinct contributions, advection of mean kinetic energy $\langle\vec{v}\rangle \cdot \vec{\nabla}\langle k\rangle$ and transport by fluctuations $\vec{\nabla} \cdot\left\langle\vec{v}^{\prime} k\right\rangle$. The latter is found to be dominant near the geometrical center where both the mean flow and the gradient of mean kinetic energy vanish. It is found positive in a sphere of radius $r=\sqrt{x^{2}+y^{2}+z^{2}} \simeq 2 \mathrm{~cm}$ so that an amount of energy nearly equivalent to the local value of $\varepsilon$ is transported outward from the center of the flow. However, the flux is very anisotropic and depends strongly on the distance to the center, as was demonstrated in a cylindrical vessel using an alternative approach [11]. Indeed, we observe advection of mean kinetic energy and turbulent transport in near equal proportions along the $x$ direction, while the turbulent flux of energy always corresponds to a loss in the $y$ direction. Although the mean properties of the turbulence vary weakly near the geometrical center, our observations indicate that the counter-rotating von Kármán flow should not be considered a quasihomogeneous turbulent flow.

\section{CONCLUSION}

We have implemented a shadow particle tracking velocimetry technique (S-PTV), using large collimated beams, to track Lagrangian tracers in a large volume around a stagnation point located at the geometrical center of a von Kármán flow. Using this dataset of Lagrangian trajectories resolving the dissipative scales of the underlying turbulence, we were able to reconstruct 3 dimensions 3 components (3D3C) ensemble-averaged maps of velocity, acceleration, and fluxes of turbulent kinetic energy, which are needed to investigate the turbulent kinetic energy budget.

A first and very general result of the present study concerns local energy dissipation, a quantity very difficult to estimate in fully turbulent flows due to the high spatial resolution needed to resolve small-scale velocity gradients. Here we demonstrated that $\left\langle\vec{a}^{\prime} \cdot \vec{v}^{\prime}\right\rangle=\langle\vec{a} \cdot \vec{v}\rangle-\langle\vec{a}\rangle \cdot\langle\vec{v}\rangle$ is a good proxy for the local dissipation $\varepsilon \simeq-\left\langle\vec{a}^{\prime} \cdot \vec{v}^{\prime}\right\rangle$ in the bulk of the flow, away from regions where energy is injected. By resolving the acceleration of the particles in a nonhomogeneous flow, it is possible to estimate the local dissipation using only one-particle statistics, without computing spatial velocity increments as is usually done when estimating dissipation from Eulerian structure functions $[9,22]$. Our approach complements the result $\langle\delta \vec{a} \cdot \delta \vec{v}\rangle=-2 \varepsilon$, derived in the context of homogeneous turbulence [23], which requires a higher particle concentration as it involves the computation of spatial increments of acceleration and velocity between two particles [24] and makes particle tracking harder.

A second result, specific to the present von Kármán flow, concerns its temporal dynamics. By a careful inspection of trajectories obtained from each movie, we demonstrated that the flow is bistable due to the presence of coherent structures attached to the walls, a consequence of the square tank geometry. Conditioning the dataset on the two states, we have reconstructed corresponding mean flows and demonstrated a rotation of $\pi / 2$ around the axis of rotation. Consequently, the present bistability is different from those observed in similar flows produced in a cylindrical vessel for which the different states mirror each other in a reflexion about the midplane $z=0[16-18,25]$. Further work is needed to fully characterize the reversals and their temporal dynamics and to test modeling approaches typically used in confined flows or more generally techniques pertaining to turbulent multi-stability such as $[16,26,27]$. For a given state, one transverse component dominates the other resulting in an impinging jet topology oriented toward the center and parallel to the $x$ or $y$ axis, depending on the bistable state. In such configuration, the flow has a stagnation point near the center which is responsible for the large anisotropy, which is most accentuated in the direction of largest strain.

By a careful investigation of the turbulent kinetic energy budget, we demonstrated the extent to which the stagnation-point topology, with only one stable direction and two unstable directions, 
is efficient at extracting energy from the mean flow. This was confirmed by our finding that the production of turbulence is nearly twice the dissipation. Production to dissipation ratios $(\mathcal{P} / \varepsilon)$ locally larger than unity are not uncommon in regions responsible for the generation of turbulence, such as the near-wall region of boundary-layer flows. However, the measurement of $\mathcal{P} / \varepsilon \simeq 2$ in the center of the vessel, several integral scales away from where the flow is forced, is unexpected.

As a consequence of such strong energy extraction, a significant part of turbulent kinetic energy, of the same order of magnitude as $\varepsilon$, is transported by turbulent fluctuations so that such a flow should be seen as strongly nonhomogeneous in space, even in the central region. Such a result is not specific to the square tank geometry and should hold when a stagnation point is present with a high level of anisotropy. This is confirmed by the data reported in Ref. [9], measured in the stagnation point of von Kármán flow produced in a cylindrical vessel, for which an estimate of the production term is found larger than dissipation [28].

\section{ACKNOWLEDGMENTS}

This work is supported by research programs ANR-13-BS09-0009 and PALSE/2013/26. Contribution from the European Project EuHIT, European High-Performance Infrastructures in Turbulence (Grant Agreement No. 312778) is also acknowledged.

[1] U. Frish, Turbulence: The Legacy of A. N. Kolmogorov (Cambridge University Press, Cambridge, UK, 1995).

[2] S. Pope, Turbulent Flows (Cambridge University Press, Cambridge, UK, 2000).

[3] H. Tennekes and J. L. Lumley, A First Course in Turbulence (MIT Press, Cambridge, MA, 1972).

[4] J.-N. Gence, Linear and nonlinear models of anisotropic turbulence, Ann. Rev. Fluid Mech. 15, 201 (1983).

[5] A. Reynolds and H. Tucker, The distortion of turbulence by general uniform irrotational strain, J. Fluid Mech. 68, 673 (1975).

[6] K. Nishino, M. Samada, K. Kasuya, and K. Torii, Turbulence statistics in the stagnation region of an axisymmetric impinging jet flow, Int. J. Heat Fluid Flow 17, 193 (1996).

[7] L. Marié and F. Daviaud, Experimental measurement of the scale-by-scale momentum transport budget in a turbulent shear flow, Phys. Fluids 16, 457 (2004).

[8] N. T. Ouellette, H. Xu, M. Bourgoin, and E. Bodenschatz, Small-scale anisotropy in Lagrangian turbulence, New J. Phys. 8, 102 (2006).

[9] G. A. Voth, A. L. Porta, A. Crawford, J. Alexander, and E. Bodenschatz, Measurement of particle accelerations in fully developed turbulence, J. Fluid Mech. 469, 121 (2002).

[10] N. Machicoane, J. Bonaventure, and R. Volk, Melting dynamics of large ice balls in a turbulent swirling flow, Phys. Fluids 25, 125101 (2013).

[11] D. Kuzzay, D. Faranda, and B. Dubrulle, Global vs local energy dissipation: The energy cycle of the turbulent von Kármán flow, Phys. Fluids 27, 075105 (2015).

[12] G. Zocchi, P. Tabeling, J. Maurer, and H. Willaime, Measurement of the scaling of the dissipation at high Reynolds numbers, Phys. Rev. E 50, 3693 (1994).

[13] P. Huck, N. Machicoane, and R. Volk, A cost-efficient shadow particle tracking velocimetry setup suitable for tracking small objects in a large volume, Proc. IUTAM 20, 175 (2017).

[14] R. Volk, N. Mordant, G. Verhille, and J.-F. Pinton, Laser Doppler measurement of inertial particle and bubble accelerations in turbulence, EPL (Europhys. Lett.) 81, 34002 (2007).

[15] P. K. Yeung and S. B. Pope, Lagrangian statistics from direct numerical simulations of isotropic turbulence, J. Fluid Mech. 207, 531 (1989).

[16] A. de la Torre and J. Burguete, Slow Dynamics in a Turbulent Von Kármán Swirling Flow, Phys. Rev. Lett. 99, 054101 (2007). 
[17] M. López-Caballero and J. Burguete, Inverse Cascades Sustained by the Transfer Rate of Angular Momentum in a 3d Turbulent Flow, Phys. Rev. Lett. 110, 124501 (2013).

[18] F. Ravelet, L. Marié, A. Chiffaudel, and F. Daviaud, Multistability and Memory Effect in a Highly Turbulent Flow: Experimental Evidence for a Global Bifurcation, Phys. Rev. Lett. 93, 164501 (2004).

[19] C. Cambon and J. Scott, Linear and nonlinear models of anisotropic turbulence, Ann. Rev. Fluid Mech. 31, 1 (1999).

[20] J. Hunt and D. Carruthers, Rapid distorsion theory and the problem of turbulence, J. Fluid Mech. 212, 497 (1990).

[21] M. M. Rogers and R. D. Moser, Direct simulation of a self-similar turbulent mixing layer, Physics of Fluids 6, 903 (1994).

[22] H. J. Hussein, S. P. Capp, and W. K. George, Velocity measurements in a high-Reynolds-number, momentum-conserving, axisymmetric, turbulent jet, J. Fluid Mech. 258, 31 (1994).

[23] R. J. Hill, Opportunities for use of exact statistical equations, J. Turbulence 7, N43 (2006).

[24] N. T. Ouellette, H. Xu, M. Bourgoin, and E. Bodenschatz, An experimental study of turbulent relative dispersion models, New J. Phys. 8, 109 (2006).

[25] O. Liot and J. Burguete, Bifurcation induced by the aspect ratio in a turbulent von Kármán swirling flow, Phys. Rev. E 95, 013101 (2017).

[26] N. Machicoane, M. López-Caballero, L. Fiabane, J. F. Pinton, M. Bourgoin, J. Burguete, and R. Volk, Stochastic dynamics of particles trapped in turbulent flows, Phys. Rev. E 93, 023118 (2016).

[27] G. Rigas, A. S. Morgans, R. D. Brackston, and J. F. Morrison, Diffusive dynamics and stochastic models of turbulent axisymmetric wakes, J. Fluid Mech. 778, R2 (2015).

[28] Voth et al., Performed Lagrangian measurements at the stagnation point of a von Kármán flow produced in a cylindrical container [9]. They found the flow anisotropy is $u_{x}^{\prime} \simeq u_{y}^{\prime} \simeq 1.54 u_{z}^{\prime}$, and reported the mean gradient tensor is almost diagonal with $\partial\left\langle v_{z}\right\rangle / \partial z \simeq u^{\prime} / L_{g}, L_{g}=0.0492 \mathrm{~m}$, and $u^{\prime 2}=\left(u_{x}^{\prime 2}+u_{y}^{\prime 2}+\right.$ $\left.u_{z}^{\prime 2}\right) / 3 \simeq 1.9 u_{z}^{\prime 2}$. This leads to a production term $\mathcal{P} \simeq 1.37 u_{z}^{\prime 2} \partial\left\langle v_{z}\right\rangle / \partial z \simeq 0.72 u^{\prime 3} / L_{g}$ slightly larger than the local value of $\varepsilon=u^{\prime 3} / L, L=0.073 \mathrm{~cm}$, estimated using the second-order velocity structure function. 